\title{
Application of diffusion approximation in quantitative photoacoustic tomography in the presence of low-scattering regions
}

\author{
Niko Hänninen ${ }^{\mathrm{a}, *}$, Aki Pulkkinen $^{\mathrm{a}}$, Aleksi Leino ${ }^{\mathrm{a}}$, Tanja Tarvainen ${ }^{\mathrm{a}, \mathrm{b}}$ \\ ${ }^{a}$ Department of Applied Physics, University of Eastern Finland, P.O. Box 1627, 70211 Kuopio, Finland \\ ${ }^{b}$ Department of Computer Science, University College London, Gower Street, London, WC1E 6BT, UK
}

\begin{abstract}
In quantitative photoacoustic tomography, the aim is to reconstruct distributions of optical parameters of an imaged target from an initial pressure distribution obtained from ultrasound measurements. In order to obtain accurate and quantitative information on the optical parameters, modeling light transport in the target is required. Utilizing an approximative model for light transport would be favorable to reduce the computational cost, but the modeling errors of the approximative model can result in significant errors in the reconstructions. In this work, we approach the image reconstruction problem of quantitative photoacoustic tomography in the Bayesian framework. We utilize the Bayesian approximation error method to compensate for the modeling errors between the diffusion approximation and Monte Carlo model for light transport. The approach is studied with two-dimensional numerical simulations with varying optical parameters and noise levels. The results show that Bayesian approximation error method can be used to reduce the effects of the modeling errors in quantitative photoacoustic tomography in a wide range of optical parameters.
\end{abstract}

Keywords: inverse problems, quantitative photoacoustic tomography, uncertainty quantification, Bayesian methods, model reduction, Bayesian approximation error modeling

\section{Introduction}

Photoacoustic tomography (PAT) is a hybrid imaging modality based on the photoacoustic effect. In the technique, images of an initial pressure distribution caused by absorption of an externally induced light pulse are reconstructed from boundary measurements of generated photoacoustic pressure waves [1, 2]. Optical absorption by different light absorbing molecules, chromophores, provide strong contrast which is combined with high resolution enabled by minimal scattering of the ultrasound waves. These properties in combination with non-invasiveness of the technique make PAT an attractive alternative for medical imaging and biomedical studies. PAT can be used, for example, to image soft biological tissues, such as blood vessels and

\footnotetext{
${ }^{*}$ Corresponding author

Email addresses: niko.hanninen@uef .fi (Niko Hänninen), aki.pulkkinen@uef .fi (Aki Pulkkinen), aleksi.leino@uef.fi (Aleksi Leino), tanja.tarvainen@uef .fi (Tanja Tarvainen) 
microvasculature of tumors in medical imaging and small animals in biomedical applications [1, 3,-7].

In quantitative photoacoustic tomography (QPAT), the aim is to estimate the concentration of chromophores from photoacoustic images [8]. This provides more accurate information of the imaged target, e.g. possibility to differentiate between oxygenated and non-oxygenated hemoglobin [8]. The estimation of the optical parameters is an ill-posed problem that needs to be approached in the framework of inverse problems.

Different approaches to estimate the concentrations of chromophores have been studied. The chromophore concentrations can be estimated directly from photoacoustic images obtained at multiple wavelengths, or by first reconstructing the absorption coefficients from the photoacoustic images and then computing the concentrations utilizing the absorption spectra of the known chromophores [8-15]. In order to obtain accurate reconstructions, scattering effects need to be considered [10, 16, 17]. Estimating more than one optical parameter in QPAT is generally a non-unique problem if only one illumination or wavelength is used [10, 18, 19]. However, this can be overcome by using multiple illuminations [10, 16, 19, -22]. It has also been shown that, at a single wavelength, piece-wise constant optical parameters can be recovered uniquely [23]. Recently, methods in which the optical parameters are estimated directly from the photoacoustic time-series have been proposed [24-29].

In this paper, we study the inverse problem of QPAT, i.e. the estimation of the optical parameters from the photoacoustic images, in the framework of Bayesian inverse problems. We assume that the initial pressure distribution has been reconstructed and the Grüneisen parameter, which connects the initial pressure and absorbed optical energy density, is known. In Bayesian framework, all parameters are modeled as random variables which are characterized by their probability distributions. The full solution of the inverse problem is the posterior distribution, which is formed by combining the measurements and prior information of the optical parameters with the model describing the physics of QPAT. However, computing the full posterior distribution using for example Markov Chain Monte Carlo methods is computationally too expensive. Therefore, point estimates of the posterior distribution are computed. In this work, we compute maximum a posteriori (MAP) estimate. Furthermore, the reliability of the reconstructions is evaluated using a local Gaussian approximation of the posterior distribution.

Due to the ill-posedness of the inverse problem of QPAT, modeling light transport in the medium is necessary to obtain accurate solutions. A generally accepted model for light transport in biological tissues is the radiative transfer equation (RTE) [30], which can be solved using numerical methods, such as finite element (FE) method. Furthermore, in addition to the deterministic models, light transport can be modeled using stochastic methods such as Monte Carlo [31, 32]. Utilizing the RTE and Monte Carlo method in QPAT have been studied for example in [16, 18, 20, 27, 33,-38], where they have been capable of providing accurate reconstructions of the absorption, scattering, or both. However, due to computationally expensive nature of these models, utilizing them in high dimensional tomographic inverse problems is often not feasible in practice. Although many computational challenges related to these models have been overcome by development of computational resources and numerical methods, such as usage of efficient parallel computing [39] or preconditioned Krylov subspace methods [40, 41], their utilization is still limited in tomographic imaging.

Iterative solving of a non-linear image reconstruction problem, such as computing the MAP estimate, requires repetitive solutions to the forward model and gradient of the objective function. Therefore, fast and efficient computational methods for their solution is required. In optical tomographic imaging, the computational cost can be reduced by using an approximative model 
for the light transport. Most commonly used approximation is the diffusion approximation (DA) of the RTE [30, 42]. The DA can be used to describe light propagation accurately relatively far from the light source, and when the scattering coefficient is significantly higher than the absorption coefficient. However, utilizing the DA in solution of the inverse problem, especially in areas of low scattering or near the light source, may result in significant errors in the solution of the inverse problem due to the modeling errors of the DA, see e.g. [16, 34, 43, 46].

In this work, we propose using the Bayesian approximation error (BAE) modeling for modeling of errors caused by an approximative forward model in QPAT. In the BAE approach, difference between the accurate and approximative model is approximated as a Gaussian random variable. Previously, the BAE modeling has been utilized in QPAT to reduce the modeling errors caused by coarse discretization of the forward model [47], marginalization of scattering coefficient [17] and inaccuracies due to the numerical approximations of an acoustic solver [48]. Here, we utilize the BAE modeling to compensate the modeling errors of the DA in QPAT. In order to form the statistics for the BAE approach, we use Monte Carlo model as the accurate reference model to which approximation error of the DA is formed. To our knowledge, this is the first study in which the BAE model is taught using a stochastic model. Previously modeling of errors between accurate and approximate light transport models have been studied with an application of diffuse optical tomography in [44, 49]. There it was shown that the BAE modeling can be used to compensate only for small modeling errors. However, these results cannot be directly extended to QPAT where data relays on absorbed optical energy within the target that is strongly dependent on absorption [16]. In this work, this approach is studied using a different range of optical parameters and noise levels.

The rest of paper is organized as follows. Light transport models utilized in this work are described in Section 2, and the inverse problem and the BAE approach for QPAT are described in Section 3. In Section 4, the numerical setup of the simulations is presented. Results are given in Section 5 and discussion and conclusions of the results are presented in Section 6

\section{Forward model}

The optical forward problem in QPAT is to solve the absorbed optical energy density $H$ within the target when the optical parameters and input light are given. In QPAT, light illumination and absorption generates acoustic waves which propagate through the domain. The propagation of the acoustic wave occurs multiple orders of magnitude slower than the absorption of light. Therefore, light propagation can be modeled using a time-independent model of light transport that can be used to describe instantaneous absorption of the light pulse [5].

\subsection{Radiative transfer equation}

Let us consider a domain $\Omega \subset \mathbb{R}^{d}$ with a boundary $\partial \Omega$ in dimension $d=2,3$ and let $\hat{s} \in S^{d-1}$ denote a unit vector in the direction of interest. A widely accepted model for light transport in biological tissue is the radiative transfer equation [30]. The time-independent RTE is

$$
\hat{s} \cdot \nabla \phi(r, \hat{s})+\left(\mu_{\mathrm{s}}(r)+\mu_{\mathrm{a}}(r)\right) \phi(r, \hat{s})=\mu_{\mathrm{s}}(r) \int_{S^{d-1}} \Theta\left(\hat{s} \cdot \hat{s}^{\prime}\right) \phi\left(r, \hat{s}^{\prime}\right) \mathrm{d} \hat{s}^{\prime}, \quad r \in \Omega
$$

where $r$ is the spatial position, $\mu_{\mathrm{a}}(r)$ is the optical absorption coefficient, $\mu_{\mathrm{s}}(r)$ is the optical scattering coefficient, $\phi(r, \hat{s})$ is the radiance and $\Theta\left(\hat{s} \cdot \hat{s}^{\prime}\right)$ is the scattering phase function [30, 42]. 
A typical choice for the scattering phase function is the Henyey-Greenstein scattering function which is of the form

$$
\Theta\left(\hat{s} \cdot \hat{s}^{\prime}\right)= \begin{cases}\frac{1}{2 \pi} \frac{1-g^{2}}{1+g^{2}-2 g \hat{s} \cdot \hat{s}^{\prime}}, & d=2 \\ \frac{1}{4 \pi} \frac{1-g^{2}}{\left(1+g^{2}-2 g \hat{s} \cdot \hat{s}^{\prime}\right)^{3 / 2}}, & d=3,\end{cases}
$$

where $-1<g<1$ is the scattering anisotropy parameter [50].

In QPAT, it is assumed that no photons travel in an inward direction at the boundary expect at source position $\epsilon \subset \partial \Omega$ which results in boundary condition

$$
\phi(r, \hat{s})= \begin{cases}\phi_{0}(r, \hat{s}), & r \in \epsilon, \hat{s} \cdot \hat{n}<0 \\ 0, & r \in \partial \Omega \backslash \epsilon, \hat{s} \cdot \hat{n}<0\end{cases}
$$

where $\hat{n}$ is an outward unit normal and $\phi_{0}(r, \hat{s})$ is a boundary source. Boundary condition (3) assumes that there are no reflections on the boundary $\partial \Omega$, i.e. the refractive index has the same value on both sides of the boundary. This assumption is equivalent to the imaged target being coupled efficiently using optically matched coupling medium. For radiative transfer in a medium with piece-wise constant refractive index, see e.g. [51]. The photon fluence $\Phi(r)$ is

$$
\Phi(r)=\int_{S^{n-1}} \phi(r, \hat{s}) \mathrm{d} \hat{s} .
$$

\subsection{Diffusion approximation}

In the diffusion approximation [30], the medium is assumed to be highly scattering $\left(\mu_{\mathrm{a}} \ll \mu_{\mathrm{s}}\right)$ and the radiance $\phi(r, \hat{s})$ is approximated by

$$
\phi(r, \hat{s}) \approx \frac{1}{\left|S^{d-1}\right|} \Phi(r)-\frac{d}{\left|S^{d-1}\right|} \hat{s} \cdot(\kappa \nabla \Phi(r)),
$$

where $\kappa(r)=\left(d\left(\mu_{\mathrm{a}}(r)+\mu_{\mathrm{s}}(r)\left(1-g_{1}\right)\right)\right)^{-1}$ is the diffusion coefficient and $g_{1}$ is the mean of cosine of the scattering angle. In the case of Heneye-Greenstein scattering phase function, $g_{1}=g$. Using similar approximations for the source term and phase functions, the DA can be derived

$$
-\nabla \cdot \kappa(r) \nabla \Phi(r)+\mu_{\mathrm{a}}(r) \Phi(r)=0, \quad r \in \Omega .
$$

For the DA, Robin-type boundary condition can be derived by assuming that the total inward directed photon current is zero [42]. This can be expressed as

$$
\xi_{d} \Phi(r)+\frac{1}{2} \kappa(r) \nabla \Phi(r) \cdot \hat{n}= \begin{cases}s(r) & r \in \epsilon \\ 0 & r \in \partial \Omega \backslash \epsilon\end{cases}
$$

where $s(r)$ is the inward light current on the boundary $\partial \Omega$ and $\xi_{d}$ is the dimension dependent scaling factor $\left(\xi_{2}=1 / \pi\right.$ and $\left.\xi_{3}=1 / 4\right)$.

In this work, the DA is used as an approximative model for light transport. We use the Galerkin finite element method (FEM) for the numerical approximation of the DA, and absorption, scattering and fluence are discretized using piecewise linear basis functions. For more details of the FE-approximation, see e.g. [16, 17, 24]. 


\subsection{Monte Carlo method}

In this work, we use Monte Carlo simulations as an accurate forward model to approximate the solution of the RTE. Monte Carlo is generally regarded as the 'gold standard' method for simulating light transport in the field of biomedical optics. In Monte Carlo method for light transport, fluence is simulated by tracing a large number of photons or photon packets inside a tissue model [31]. Due to the flexibility of this approach, it has been widely used to simulate the light transport in biological tissues, and numerous implementations of Monte Carlo algorithms exist [32]. While Monte Carlo model can provide any desired accuracy for the solution of the light transport problem, achieving accurate solutions can be computationally expensive due to the stochastic nature of the approach. In this work, we use the photon packet method implemented in open source software ValoMC and the associated MATLAB toolbox to perform the Monte Carlo simulations [52].

\subsection{Photoacoustic effect}

As light propagates within the medium, it is absorbed by light absorbing molecules (chromophores). Absorbed optical energy density $H$ is

$$
H(r)=\mu_{\mathrm{a}}(r) \Phi(r)
$$

where $\Phi(r)$ is the photon fluence given by the light transport model. This generates localised increases in pressure. The initial acoustic pressure distribution $p_{0}(r)$ is

$$
p_{0}(r)=G(r) H(r),
$$

where $G(r)$ denotes the photoacoustic efficiency, which describes the conversion efficiency of heat energy to pressure [1]. For soft tissues, commonly modeled acoustically as fluids, this efficiency can be expressed with the Grüneisen parameter $G$, which is a thermodynamic property of material and can be expressed as $G=\beta c_{o}^{2} / C_{p}$, where $\beta$ is the volume thermal expansivity of the fluid, $c_{0}$ is the sound speed, and $C_{p}$ is the isobaric specific heat capacity [8].

\section{Inverse problem}

In this work, the optical inverse problem of QPAT, i.e. estimation of the optical absorption and scattering parameters from photoacoustic images is studied. We assume that the acoustic inverse problem has been solved and the Grüneisen parameter is known.

Let us denote the measurements by $y=\left[H_{1}, H_{2}, \ldots, H_{M}\right] \in \mathbb{R}^{M}$, where $M$ is the number of measurements, which is the number of illuminations times the number of discretization points in the measurement grid. Further, $x=\left[\mu_{\mathrm{a}}, \mu_{\mathrm{s}}\right] \in \mathbb{R}^{2 N}$ denotes discretized parameters $\mu_{\mathrm{a}}=$ $\left[\mu_{\mathrm{a}_{1}}, \mu_{\mathrm{a}_{2}}, \ldots, \mu_{\mathrm{a}_{N}}\right] \in \mathbb{R}^{N}$ and $\mu_{\mathrm{s}}=\left[\mu_{\mathrm{s}_{1}}, \mu_{\mathrm{s}_{2}}, \ldots, \mu_{\mathrm{s}_{N}}\right] \in \mathbb{R}^{N}$ where $N$ is the number of discretization points in the parameter grid. The discretized observation model with an additive noise model is

$$
y=f_{\delta}(x)+e,
$$

where $f_{\delta}: \mathbb{R}^{2 N} \mapsto \mathbb{R}^{M}$ is the discretized forward model that is assumed to be exact within measurement precision and $e \in \mathbb{R}^{M}$ is the measurement noise. Here, the noise $e$ is the random noise of the absorbed energy density. Typically, measurement noise in the photoacoustic ultrasound 
measurements is assumed to be additive. Due to the linear acoustical modeling commonly employed in photoacoustics, additive noise in the ultrasound measurements translates as an additive noise in photoacoustic images.

In many applications the accurate model is unavailable or utilizing the accurate model is unfeasible due to high demands of computational resources. Thus, an approximative forward model $f_{h}$ is often used. Utilizing Bayesian approximation error modeling [53] this leads to an observation model

$$
\begin{aligned}
y & =f_{h}(x)+\left(f_{\delta}(x)-f_{h}(x)\right)+e \\
& =f_{h}(x)+\varepsilon(x)+e \\
& =f_{h}(x)+v
\end{aligned}
$$

where $\varepsilon(x)=f_{\delta}(x)-f_{h}(x)$ is the modeling error which describes the difference between the approximative and accurate model and $v=\varepsilon+e$ is the total error.

\subsection{Bayesian framework}

In the Bayesian approach [53], all parameters are considered as random variables. Solution of the inverse problem is the posterior probability density $\pi(x \mid y)$, which according to Bayes' formula is

$$
\pi(x \mid y)=\frac{\pi(x) \pi(y \mid x)}{\pi(y)},
$$

where $\pi(x)$ is the prior density, $\pi(y \mid x)$ is the likelihood density and $\pi(y)$ is the normalization constant. The prior density describes the prior information about the unknown $x$, and the likelihood density describes the likelihood of a specific measurement outcome with the given parameters. Probability density $\pi(y)$ is a constant for a given measurement, and therefore we can write the posterior as

$$
\pi(x \mid y) \propto \pi(x) \pi(y \mid x) .
$$

Further, if $x$ and $e$ are independent, the posterior distribution can be written in the form

$$
\pi(x \mid y) \propto \pi(x) \pi_{\text {noise }}(y-f(x)),
$$

where $\pi_{\text {noise }}$ is the probability density of the error $v$.

\subsection{Conventional error modeling}

In conventional approach, the approximative model $f_{h}$ is treated as accurate, and approximation error $\varepsilon(x)$ is ignored, i.e. $\varepsilon=0$. In this work, the unknown parameter $x$ and the noise $e$ are modeled as Gaussian distributions with

$$
x \sim \mathcal{N}\left(\eta_{x}, \Gamma_{x}\right), \quad e \sim \mathcal{N}\left(\eta_{e}, \Gamma_{e}\right) .
$$


where $\eta_{x} \in \mathbb{R}^{2 N}$ and $\eta_{e} \in \mathbb{R}^{M}$ are the means and $\Gamma_{x} \in \mathbb{R}^{2 N \times 2 N}$ and $\Gamma_{e} \in \mathbb{R}^{M \times M}$ are the covariance matrices [53]. With these models, the posterior density can be written as

$$
\pi(x \mid y) \propto \exp \left\{-\frac{1}{2}\left(y-f_{h}(x)-\eta_{e}\right)^{\mathrm{T}} \Gamma_{e}^{-1}\left(y-f_{h}(x)-\eta_{e}\right)-\frac{1}{2}\left(x-\eta_{x}\right)^{\mathrm{T}} \Gamma_{x}^{-1}\left(x-\eta_{x}\right)\right\} .
$$

Computing the full posterior distribution is typically computationally too expensive in practical tomographic imaging problems. Therefore, point estimates are considered. In this work, the MAP estimate is considered. It can be obtained by minimizing the negative of the exponent term of the posterior distribution

$$
\begin{aligned}
\hat{x} & =\underset{x}{\arg \max } \pi(x \mid y) \\
& =\underset{x}{\arg \min }\left\{\frac{1}{2}\left\|L_{e}\left(y-f_{h}(x)-\eta_{e}\right)\right\|^{2}+\frac{1}{2}\left\|L_{x}\left(x-\eta_{x}\right)\right\|^{2}\right\},
\end{aligned}
$$

where $\hat{x}$ is the MAP estimate and $\Gamma_{x}^{-1}=L_{x}^{\mathrm{T}} L_{x}$ and $\Gamma_{e}^{-1}=L_{e}^{\mathrm{T}} L_{e}$ are the Cholesky decompositions of the inverse of the covariance matrices. Here, we refer to the solution of 16 as the MAP estimate with the conventional error model (MAP-CEM).

\subsection{Bayesian approximation error modeling}

In the Bayesian approximation error (BAE) modeling [53], the approximation error $\varepsilon(x)$ and total error $v$ are modeled as Gaussian distributed

$$
\varepsilon \sim \mathcal{N}\left(\eta_{\varepsilon}, \Gamma_{\varepsilon}\right), \quad v \sim \mathcal{N}\left(\eta_{v}, \Gamma_{v}\right),
$$

where $\eta_{\varepsilon}$ is the mean and $\Gamma_{\varepsilon}$ is the covariance of the approximation error, and $\eta_{v}=\eta_{e}+\eta_{\varepsilon}$ and $\Gamma_{v}=\Gamma_{e}+\Gamma_{\varepsilon}$. Using Gaussian distributed modeling error is a highly approximate model. The same approximation has been, however, utilized succesfully in numerous studies applying the BAE, for example in [17, 47, 48, 54-57]. If the mutual dependence of $x$ and $\varepsilon$ is ignored, the posterior density becomes

$$
\pi(x \mid y) \propto\left\{-\frac{1}{2}\left(y-f_{h}(x)-\eta_{v}\right)^{\mathrm{T}} \Gamma_{v}^{-1}\left(y-f_{h}(x)-\eta_{v}\right)-\frac{1}{2}\left(x-\eta_{x}\right)^{\mathrm{T}} \Gamma_{x}^{-1}\left(x-\eta_{x}\right)\right\} .
$$

The MAP estimate using the BAE is obtained as

$$
\hat{x}=\underset{x}{\arg \min }\left\{\frac{1}{2}\left\|L_{\nu}\left(y-f_{h}(x)-\eta_{\nu}\right)^{\mathrm{T}}\right\|^{2}+\frac{1}{2}\left\|L_{x}\left(x-\eta_{x}\right)\right\|^{2}\right\},
$$

where $\Gamma_{v}^{-1}=L_{v}^{\mathrm{T}} L_{v}$. In this paper, we refer to the solution of $(18)$ as the MAP estimate obtained with an enhanced error model (MAP-EEM).

In order to apply the approximation error statistics in the solution of the inverse problem, the statistics needs to be determined. In practice, the approximation error can be approximated by investigating samples of the errors between the accurate model and the approximative model as follows [48]. First, a set of samples $\left\{x^{(\ell)}, \ell=1, \ldots, L\right\}$ are drawn from the prior distribution of the optical parameters. Next, the forward problem is solved using the accurate and approximative 
models. Result is a set of forward solutions $\left\{f_{\delta}\left(x^{(\ell)}\right)\right\}$ and $\left\{f_{h}\left(x^{(\ell)}\right)\right\}$. Samples of the approximation error can then be computed as

$$
\varepsilon^{(\ell)}=f_{\delta}\left(x^{(\ell)}\right)-f_{h}\left(x^{(\ell)}\right)
$$

and the mean and the covariance of the BAE can be approximated as

$$
\begin{aligned}
\eta_{\varepsilon} & =\frac{1}{L} \sum_{\ell=1}^{L} \varepsilon^{(\ell)} \\
\Gamma_{\varepsilon} & =\frac{1}{L-1} \sum_{\ell=1}^{L} \varepsilon^{(\ell)} \varepsilon^{(\ell)^{\mathrm{T}}}-\eta_{\varepsilon} \eta_{\varepsilon}^{\mathrm{T}} .
\end{aligned}
$$

While computing the approximation error statistics can be time consuming, it is only required once for a specific geometry, illumination configurations, and prior information. Thus, it can be done off-line before the measurements and solving the inverse problem.

\subsection{Evaluating credibility}

In addition to point estimates for the unknown parameters, Bayesian framework can be used to evaluate the reliability of the estimates using credibility intervals. In this work, we estimate the reliability of the estimates similarly as in [24, 47]. In this approach, the forward model is approximated using the first order Taylor series. Using this approximation, the posterior distribution can be approximated locally as a Gaussian distribution

$$
x \mid y \sim \mathcal{N}(\hat{\eta}, \hat{\Gamma})
$$

where $\hat{\eta}=\hat{x}$ is the MAP estimate and

$$
\hat{\Gamma}=\left(J(\hat{x})^{\mathrm{T}} \Gamma^{-1} J(\hat{x})+\Gamma_{x}^{-1}\right)^{-1}
$$

is the covariance matrix, where $J(\hat{x})$ is the Jacobian matrix of $f_{h}(x)$ evaluated at point $\hat{x}$ and $\Gamma=\Gamma_{e}$ for MAP-CEM and $\Gamma=\Gamma_{v}$ for MAP-EEM estimate.

For a Gaussian distribution, credibility intervals can be computed from the standard deviation (SD) of the distribution. We compute these credibility intervals $\left[\eta_{\hat{x}}-p \sigma_{\hat{x}}, \eta_{\hat{x}}+p \sigma_{\hat{x}}\right]$ with different values of $p$, where $\eta_{\hat{x}_{j}}$ and $\sigma_{\hat{x}_{j}}$ are the mean and the standard deviation of $\hat{x}_{j}$. The standard deviation of the parameter $\hat{x}_{j}$ is obtained from the diagonal values of the covariance matrix of the posterior approximation

$$
\sigma_{\hat{x}_{j}}=\sqrt{\hat{\Gamma}(j, j)}
$$

For example, for a true Gaussian posterior distribution, the true value of the parameter $x_{j}$ lies with probability of $99.7 \%$ in the interval $\left[\eta_{\hat{x}_{j}}-3 \sigma_{\hat{x}_{j}}, \eta_{\hat{x}_{j}}+3 \sigma_{\hat{x}_{j}}\right]$.

\section{Simulation studies}

We studied compensation of the modeling errors of the DA in QPAT by the BAE modeling with two-dimensional (2D) simulations in a $6 \mathrm{~mm} \times 4 \mathrm{~mm}$ rectangular domain. Feasibility 
Table 1: Ranges of absorption $\mu_{\mathrm{a}}\left(\mathrm{mm}^{-1}\right)$ and scattering $\mu_{\mathrm{s}}\left(\mathrm{mm}^{-1}\right)$ coefficients with different scaling factors.

\begin{tabular}{ccc}
\hline Scaling factor & $\mu_{\mathrm{a}}$ range & $\mu_{\mathrm{s}}$ range \\
\hline 2 & $0.14-0.8$ & $2-24$ \\
1 & $0.07-0.4$ & $1-12$ \\
0.5 & $0.035-0.2$ & $0.5-6$ \\
0.25 & $0.0175-0.1$ & $0.25-3$ \\
0.1 & $0.007-0.04$ & $0.1-1.2$ \\
\hline
\end{tabular}

Table 2: Discretizations used in the study: number of elements $N_{e}$ and number of nodes $N_{n}$.

\begin{tabular}{lccc}
\hline & Mesh & $N_{e}$ & $N_{n}$ \\
\hline Data simulation & $\mathcal{H}_{s}$ & 48310 & 95818 \\
Coarse mesh, basis for the optical parameters & $\mathcal{H}_{h}$ & 1024 & 1932 \\
Fine mesh & $\mathcal{H}_{\delta}$ & 15685 & 30912 \\
\hline
\end{tabular}

of the BAE approach was investigated in a study, where a range of absorption and scattering coefficients and the amount of measurement noise were varied in a target with smooth inclusions. Furthermore, another target with blood-vessel mimicking inclusions with optical parameters in the scale of blood and fat tissue was studied [58-60].

For the first target, the different ranges of optical parameters were obtained by scaling a reference range of the optical parameters. A reference range, i.e. the minimum and maximum values of the optical parameters was chosen to be $0.07-0.4 \mathrm{~mm}^{-1}$ for the absorption coefficient and $1-12 \mathrm{~mm}^{-1}$ for the scattering coefficient. This scale of the parameters corresponds to the values which can be found in the biological tissues, and where the DA is considered to be accurate [58-60]. Then, to obtain the different ranges for the studies, the reference range was scaled with a constant scaling factor ranging from 0.1 to 2 . The ranges for the optical parameters with different scaling parameters are given in Table 1 . In all simulations, the scattering anisotropy parameter $g=0.8$ was used. The simulations were performed in MATLAB (R2017b MathWorks Inc., Natick, MA).

\subsection{Data simulation}

To simulate data, the rectangular domain was illuminated from four sides independently creating a data vector based on these four different illuminations. On each illumination, the entire boundary on one side of the target was illuminated, i.e. acted as a light source. The photon fluence was simulated using a Monte Carlo method in a piecewise constant triangular discretization $\mathcal{H}_{s}$ with $10^{9}$ photon packets and light source with a cosinic angular shape using ValoMC software and MATLAB toolbox [52]. Then, the absorbed optical energy density was computed using Eq. (8). To avoid making an inverse crime, the simulated data was interpolated to a piecewise linear discretization $\mathcal{H}_{h}$ which was the data space of the inverse problem. Then, random Gaussian noise with zero mean was added to the simulated optical energy density data. The simulations with the smooth inclusions were studied with three different standard deviations of the noise: $0.1 \%, 1 \%$ and $5 \%$ of the maximum value of the simulated data. In the simulation with blood-vessel mimicking inclusions, the standard deviation of the noise was $1 \%$ of the maximum value of the simulated data. The number of the elements and nodes of the discretizations utilized in the simulations are given in Table 2 


\subsection{Inverse problem}

The inverse problem was solved using the methodology described in Section 2. Two MAP estimates were studied: a MAP estimate with the conventional error model (MAP-CEM) which was obtained by minimizing (16) and a MAP estimate with the enhanced error model (MAPEEM) which was obtained by minimizing (18). In both cases, the fluence was solved using the DA and represented in a piece-wise linear basis in the coarse discretization $\mathcal{H}_{h}$, i.e. the number of measurements was $M=4 \times N_{n}$, where 4 arises from the number of illuminations, and $N_{n}$ is the number of nodes in the grid $\mathcal{H}_{h}$. The unknown absorption and scattering parameters were presented in piece-wise linear bases in $\mathcal{H}_{h}$, i.e. the number of discretization points in the parameter grid was $N=N_{n}$, where $N_{n}$ is the number of nodes in the grid $\mathcal{H}_{h}$. In the CEM approach, the approximation error $\varepsilon$ was ignored. In the EEM approach the approximation error was approximated as a Gaussian distribution (details given in Section 4.4).

In both approaches, the noise was modeled as Gaussian distributed using the known noise level, i.e. with mean $\eta_{e}=0$ and constant standard deviation of $0.1 \%, 1 \%$ or $5 \%$ of the maximum value of the full data vector. As a prior, Ornstein-Uhlenbeck prior model was used (details given in Section 4.3.

The estimated parameters were scaled to ensure the numerical stability of the reconstruction algorithm similarly as in [16, 47]. The minimization problems (16) and (18) were solved using a Gauss-Newton method utilizing a line-search algorithm with positivity constrain for determining the step size parameter. An initial value for the Gauss-Newton algorithm was chosen to be the mean of the prior. Solution was assumed to be converged, when the total change in the norm which was minimized was smaller than $10^{-3}$ in three consecutive iterations.

In order to evaluate the reliability of the reconstructed images, the credibility intervals were approximated as described in Section 3.4. Further, results were compared visually and by computing relative errors of the estimated parameters by

$$
E_{\mu_{\mathrm{a}}}=100 \% \cdot \frac{\left\|\hat{\mu}_{\mathrm{a}}-\mu_{\mathrm{a}}^{\mathrm{sim}}\right\|}{\left\|\mu_{\mathrm{a}}^{\mathrm{sim}}\right\|}, \quad E_{\mu_{\mathrm{s}}}=100 \% \cdot \frac{\left\|\hat{\mu}_{\mathrm{s}}-\mu_{\mathrm{s}}^{\mathrm{sim}}\right\|}{\left\|\mu_{\mathrm{s}}^{\mathrm{sim}}\right\|},
$$

where $\mu_{\mathrm{a}}^{\text {sim }}$ and $\mu_{\mathrm{s}}^{\text {sim }}$ are the simulated distributions of the absorption and scattering coefficients, respectively, $\hat{\mu}_{\mathrm{a}}$ and $\hat{\mu}_{\mathrm{s}}$ are the estimated distributions interpolated to the simulation grid, and the norm is the Euclidean norm.

\subsection{Prior model}

In this study, Ornstein-Uhlenbeck prior model was used [61]. Ornstein-Uhlenbeck prior is a Gaussian distribution with the covariance matrix defined as

$$
\Gamma_{\mu}=\sigma_{\mu}^{2} \Xi
$$

where $\sigma_{\mu}$ is the standard deviation of the prior distribution, $\mu$ is either $\mu_{\mathrm{a}}$ or $\mu_{\mathrm{s}}$ and $\Xi$ is defined by its elements

$$
\Xi(i, j)=\exp \left(-\left\|r_{i}-r_{j}\right\| / l\right),
$$

where $i$ and $j$ denote the row and column indices of the matrix, $r_{i}$ and $r_{j}$ denotes the grid node coordinates and $l$ is the characteristic length scale parameter. The length scale parameter can be chosen such that we assume significant correlation within distance $l$. The full prior model 
utilized in this work was then

$$
\eta_{x}=\left(\begin{array}{l}
\eta_{\mu_{\mathrm{a}}} \\
\eta_{\mu_{\mathrm{s}}}
\end{array}\right), \quad \Gamma_{x}=\left(\begin{array}{cc}
\Gamma_{\mu_{\mathrm{a}}} & 0 \\
0 & \Gamma_{\mu_{\mathrm{s}}}
\end{array}\right) .
$$

In the reconstructions, the absorption values of the target were assumed to be within interval $\left[0, \max \left(\mu_{\mathrm{a}}^{\mathrm{sim}}\right)\right]$, where $\mu_{\mathrm{a}}^{\mathrm{sim}}$ is the true simulated absorption distribution. The mean of the prior distribution was chosen to be the mean of that interval, and the standard deviation was chosen such that the interval is within one standard deviation from the mean. For the scattering, the prior parameters were chosen in same fashion based on $\mu_{\mathrm{s}}^{\mathrm{sim}}$. The mean and standard deviation of the priors for the absorption and scattering used in this work are given in Table 3 The characteristic length scale $l=0.5 \mathrm{~mm}$ was used in all of the simulations.

\subsection{Approximation error statistics}

The statistics of approximation error was computed as described in Section 3.3 For each different range of the optical parameters, the statistics of the approximation error were computed separately as follows. 6000 samples $\left\{x^{(\ell)}\right\}$ were drawn from the training distributions for the absorption and scattering with the prior parameters described in Table 3 and with the characteristic length scale of $l=0.5 \mathrm{~mm}$. The standard deviation of the training distributions utilized in the computation of the BAE statistics were chosen to be smaller than the standard deviations of the prior distributions utilized in the reconstructions. We observed that using a smaller standard deviation in the training distribution resulted in more effective alleviation of the modeling errors with the BAE approach. This is due to the nonlinear nature of the approximation error. For training distributions with large standard deviations, the Gaussian approximation employed can fail to model the statistical nature of the error effectively. In a rare occasion that negative parameters were drawn (with probability less than $0.15 \%$ considering the mean and standard deviation), they were set to the value $10^{-6}$ in order to keep the model physical.

Solution to the forward problem was solved using two different methods: Monte Carlo method, which was considered as an accurate model, and FE-approximation of the DA which was considered as an approximative model. The Monte Carlo method was solved in piece-wise constant discretization $\mathcal{H}_{\delta}$ using $10^{8}$ photon packets and interpolated to the piece-wise linear discretization $\mathcal{H}_{h}$. The FE-approximation of DA was solved in a piece-wise linear discretization $\mathcal{H}_{h}$. While the discretization $\mathcal{H}_{h}$ can be considered accurate enough for the DA in this domain, the finer discretization for Monte Carlo solution was used to minimize the interpolation errors between the piece-wise constant and piece-wise linear discretizations. These resulted in fluences $\left\{\Phi_{\delta}\left(x^{(\ell)}\right)\right\}$ and $\left\{\Phi_{h}\left(x^{(\ell)}\right)\right\}$, respectively. The absorbed optical energies $\left\{H_{\delta}\left(x^{(\ell)}\right)\right\}$ and $\left\{H_{h}\left(x^{(\ell)}\right)\right\}$ were computed using Eq. (8). Samples of the approximation error were computed using these absorbed optical energy densities, and the mean and the covariance of the approximation error were computed using (20) and (21). Due to numerical errors in the Monte Carlo computations, a small number of samples $(<20)$ were not computed properly and were discarded from the final computations.

Figure 1 shows the structure of the total error covariance matrix $\Gamma_{v}$ of the enhanced error model with different noise levels simulated for the reference parameter range. As it can be seen, the total error covariance matrices show a complicated structure. On the other hand, the noise covariance matrix of conventional error model is diagonal. This indicates that one can expect different solutions of the inverse problem depending on which noise model is utilized. Further, the spatial distributions of the diagonal values of the total error covariance matrices show that 

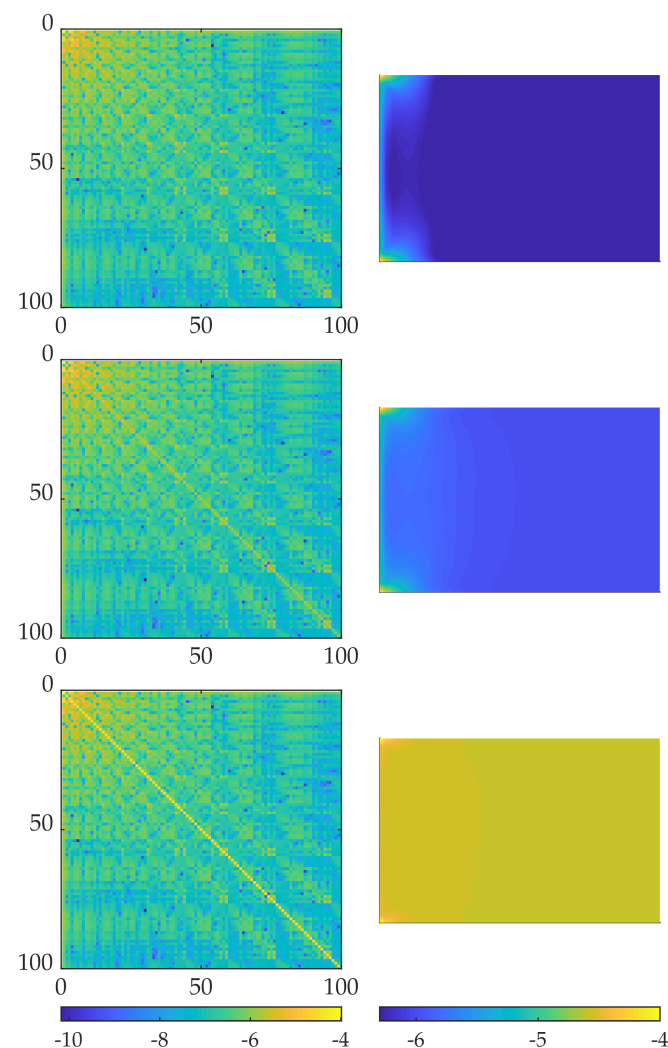

Figure 1: Logarithm of the absolute value of the total error covariance matrix $\log _{10}\left|\Gamma_{v}\right|$ corresponding to first 100 data points (left column) and logarithm of the diagonal of the total error covariance matrix $\log _{10}\left(\operatorname{diag}\left(\Gamma_{v}\right)\right)$ plotted in the data grid (right column) of the EEM. Results are shown with $0.1 \%$ noise (top row), $1 \%$ noise (second row) and 5\% noise (bottom row) with optical parameters in the reference range (corresponding to a scaling factor of 1 in Table 1 and one illumination (left side of the domain).

Table 3: Prior parameters used in the reconstructions and computation of the BAE statistics: the mean of the absorption $\eta_{\mu_{a}}\left(\mathrm{~mm}^{-1}\right)$, the mean of the scattering $\eta_{\mu_{\mathrm{s}}}\left(\mathrm{mm}^{-1}\right)$, the standard deviation of the absorption $\sigma_{\mu_{a}}\left(\mathrm{~mm}^{-1}\right)$ and the standard deviation of the scattering $\sigma_{\mu_{s}}\left(\mathrm{~mm}^{-1}\right)$.

\begin{tabular}{ccccccccc}
\hline & & & \multicolumn{4}{c}{ Reconstructions } & \multicolumn{2}{c}{ BAE statistics } \\
\hline Scaling factor & $\mu_{\mathrm{a}}$ range & $\mu_{\mathrm{s}}$ range & $\eta_{\mu_{\mathrm{a}}}$ & $\eta_{\mu_{\mathrm{s}}}$ & $\sigma_{\mu_{\mathrm{a}}}$ & $\sigma_{\mu_{\mathrm{s}}}$ & $\sigma_{\mu_{\mathrm{a}}}$ & $\sigma_{\mu_{\mathrm{s}}}$ \\
\hline 2 & $0.14-0.8$ & $2-24$ & 0.4 & 12 & 0.4 & 12 & 0.13 & 4 \\
1 & $0.07-0.4$ & $1-12$ & 0.2 & 6 & 0.2 & 6 & 0.067 & 2 \\
0.5 & $0.035-0.2$ & $0.5-6$ & 0.1 & 3 & 0.1 & 3 & 0.034 & 1 \\
0.25 & $0.0175-0.1$ & $0.25-3$ & 0.05 & 1.5 & 0.05 & 1.5 & 0.017 & 0.5 \\
0.1 & $0.007-0.04$ & $0.1-1.2$ & 0.02 & 0.6 & 0.02 & 0.6 & 0.0067 & 0.2 \\
\hline
\end{tabular}

the approximation errors are more significant near the illumination, and with high noise level the approximation errors become less significant compared to the random noise. 


\section{Results}

\subsection{Varying optical parameters with $1 \%$ noise}

The simulated (true) optical parameters and the MAP estimates obtained with conventional error model (MAP-CEM) and enhanced error model (MAP-EEM) with 1\% random noise and different ranges of scattering coefficient are shown in Figure 2 and with different ranges of absorption and scattering coefficients are shown in Figure 3. By visual inspection, the absorption estimates are qualitatively more accurate compared to the scattering estimates, which has been evident also in other QPAT studies [16, 20, 35]. Comparing the MAP-CEM and MAP-EEM estimates, the absorption estimates resemble each other but there are some differences especially in the inclusions near the center of the domain. When looking at the MAP-CEM scattering estimates, significant artefacts especially near the boundary of the domain can be seen. These are evident in the domains with low scattering (leftmost columns) and are due to the fact that the DA is not a valid approximation in the vicinity of the light source nor within low-scattering medium. The BAE modeling is capable for compensating these errors and especially the quality of the scattering reconstructions is improved.

The MAP-estimates with credibility intervals along the cross-section through the domain are shown in Figure 4 for the reference range of the optical parameters. In the cross-section, the differences between the MAP-CEM and MAP-EEM estimates and their credibility intervals are clearly visible. In the MAP-CEM absorption estimate, there is a systematic error and estimates are higher than the true parameters in the whole cross-section. Further, the true absorption values do not lie within the principal support of the credibility intervals in the whole domain. In the MAP-EEM estimates, the estimated absorption and scattering parameters are closer to the true values. Further, the true absorption and scattering values are within the credibility intervals in the whole domain. The same properties of the MAP estimates can also be seen in the marginal densities of the posterior distributions in two points inside the domain presented in Figure 5. As it can be seen, in addition to MAP-EEM absorption estimates being closer to the true values, the higher uncertainty of the estimates results in wider credibility intervals of the estimates. This is especially evident in the point near the boundary of the domain. Furthermore, in contrast to the MAP-CEM estimates, the true values lie within the credibility interval of the MAP-EEM estimates.

\subsection{Effect of measurement noise}

The simulations described in the previous subsection were repeated with different amount of additive noise. The MAP estimates were computed for all targets from data simulated with noise of levels $0.1 \%, 1 \%$, and $5 \%$ of the maximum amplitude of the simulated data. Relative errors of the MAP-CEM and MAP-EEM estimates computed of these studies with different ranges of the optical parameters and noise levels are given in Tables 4,5 and 6, respectively. Furthermore, they are visualized in Figures 6,7 and 8 .

As evident, with $0.1 \%$ and $1 \%$ noise the relative errors of the MAP-EEM scattering estimates are consistently smaller compared to MAP-CEM estimates, and the difference between the errors increases when the scattering decreases. Similarly, the relative errors of the MAP-EEM absorption estimates are lower than the errors of the MAP-CEM estimates in general. However, in situation where absorption coefficient is in the reference range and the values of scattering coefficient are small, the difference between the relative errors are very small, and in one case the MAP-CEM estimate is more accurate than the MAP-EEM estimate. In those situations, the scattering coefficient is not significantly higher than the absorption coefficient anymore, and therefore 

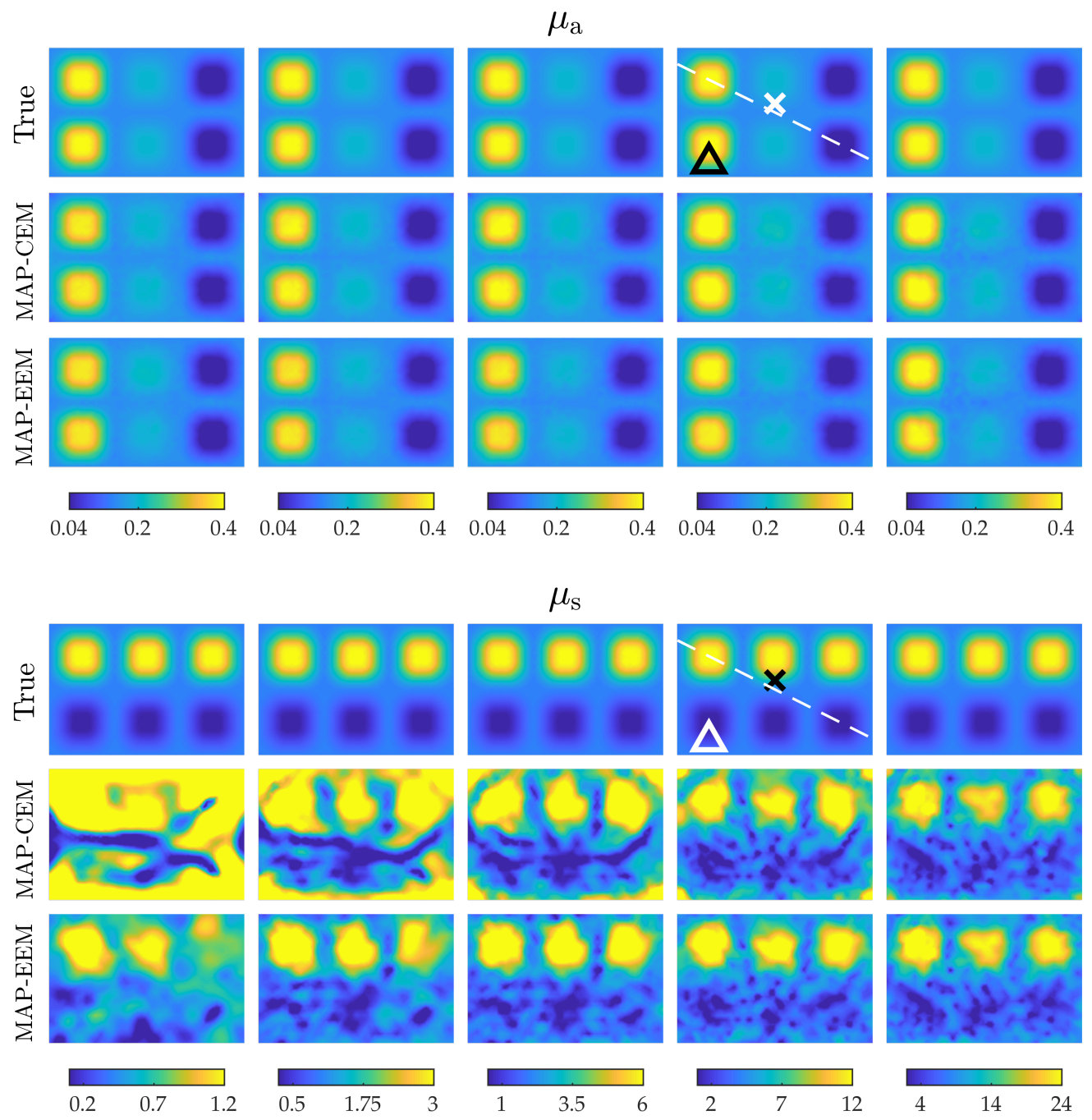

Figure 2: True target (first row), MAP estimate with conventional error model (second row) and MAP estimates with enhanced error model (third row) with varying ranges of scattering coefficient. Absorption coefficient $\mu_{\mathrm{a}}$ (top) and scattering coefficient $\mu_{\mathrm{s}}$ (bottom). Units in the colorbar are in $\mathrm{mm}^{-1}$. White dashed line (fourth column) indicates the cross-section in which the credibility intervals are presented, and $\times$ and $\Delta$ indicate the points in which the marginal densities are presented. Results shown are based on data with $1 \%$ noise. 

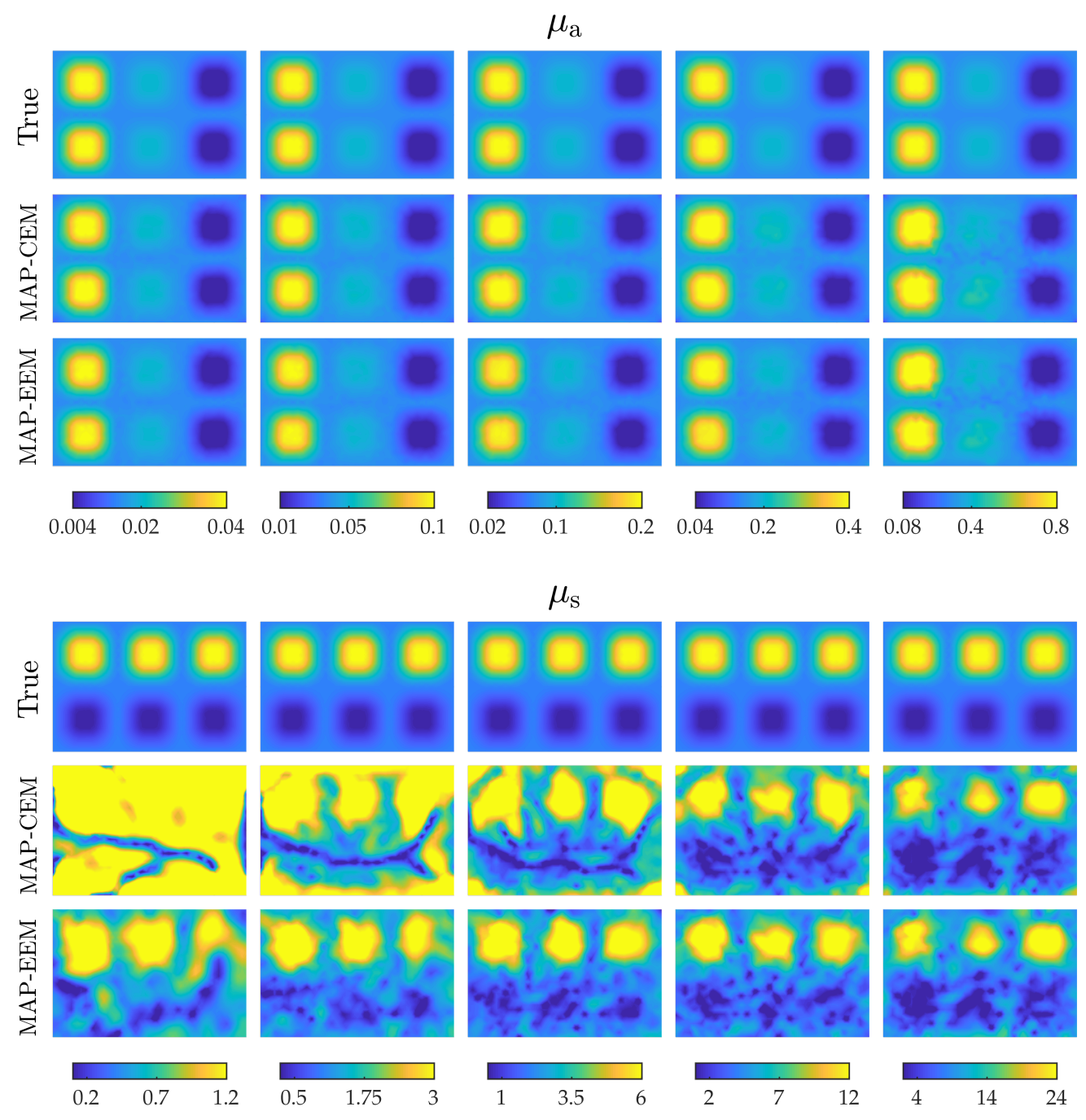

Figure 3: True target (first row), MAP estimate with conventional error model (second row) and MAP estimates with enhanced error model (third row) with varying ranges of absorption and scattering coefficients. Absorption coefficient $\mu_{\mathrm{a}}$ (top) and scattering coefficient $\mu_{\mathrm{s}}$ (bottom). Units in the colorbar are in $\mathrm{mm}^{-1}$. Results shown are based on data with $1 \%$ noise. 

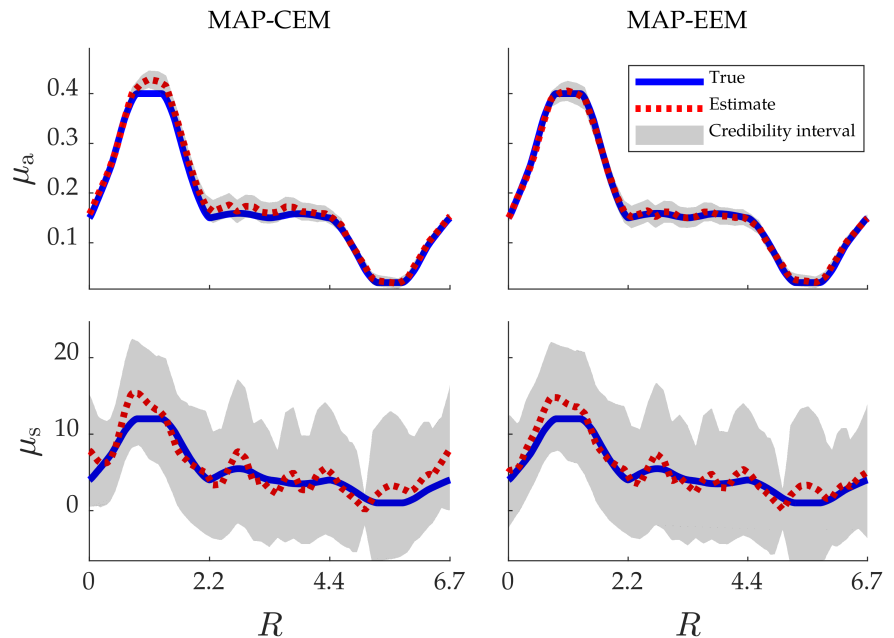

Figure 4: MAP-CEM reconstructions (first column) and MAP-EEM reconstructions (second column) with credibility intervals of the simulations with optical parameters in the reference range along the cross-section shown in Figure 2 Solid blue line is the true value and dotted red line is MAP estimate and gray area covers the credibility interval [ $\hat{\mu}-$ $\left.3 \sigma_{\hat{\mu}}, \hat{\mu}+3 \sigma_{\hat{\mu}}\right]$. First row presents the absorption $\mu_{\mathrm{a}}\left(\mathrm{mm}^{-1}\right)$ and second row the scattering $\mu_{\mathrm{s}}\left(\mathrm{mm}^{-1}\right)$. Horizontal axis $R$ presents the distance from the upper left end point of the cross-section. Results shown are based on data with $1 \%$ noise.
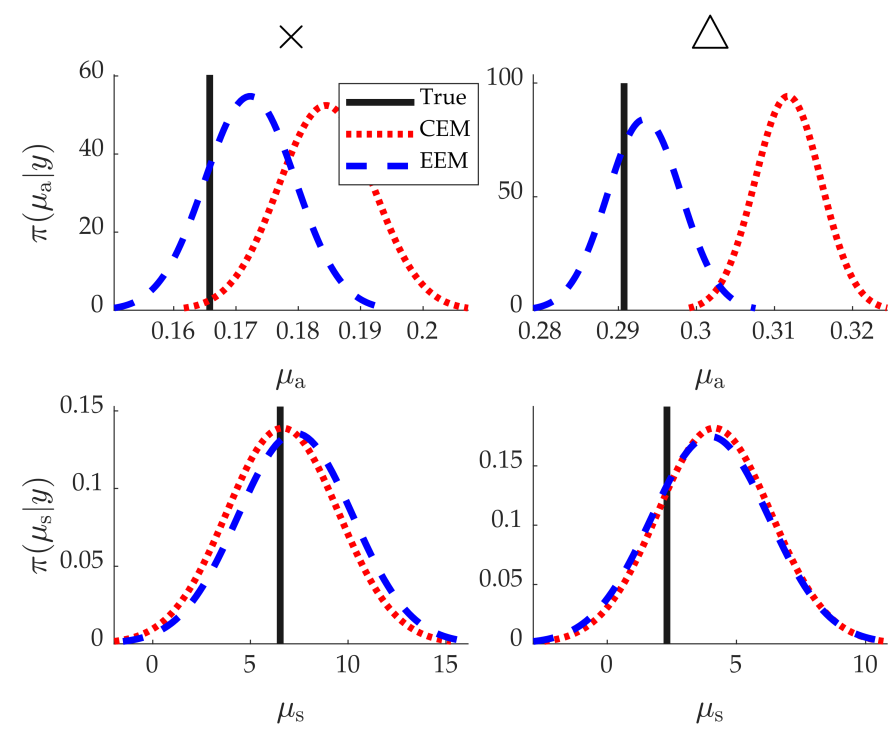

Figure 5: Marginal probability densities of the posterior distributions. True value (solid vertical line), the approximation of the posterior distribution of MAP-CEM reconstructions (red dotted line) and MAP-EEM reconstructions (blue dashed line). The first row presented the absorption $\mu_{\mathrm{a}}\left(\mathrm{mm}^{-1}\right)$ and second row the scattering $\mu_{\mathrm{s}}\left(\mathrm{mm}^{-1}\right)$. First column presents the results in the point marked with $\times$ and second row with $\Delta$ in the Figure 2 Results shown are based on data with $1 \%$ noise. 
Table 4: Relative errors of the MAP-CEM and MAP-EEM estimates $E_{\mu_{\mathrm{a}}}(\%)$ and $E_{\mu_{\mathrm{s}}}(\%)$ with different ranges of absorption $\mu_{\mathrm{a}}\left(\mathrm{mm}^{-1}\right)$ and scattering $\mu_{\mathrm{s}}\left(\mathrm{mm}^{-1}\right)$ coefficients with $0.1 \%$ noise.

\begin{tabular}{cccccc}
\hline & & \multicolumn{2}{c}{ MAP-CEM } & \multicolumn{2}{c}{ MAP-EEM } \\
\hline$\mu_{\mathrm{a}}$ range & $\mu_{\mathrm{s}}$ range & $E_{\mu_{\mathrm{a}}}$ & $E_{\mu_{\mathrm{s}}}$ & $E_{\mu_{\mathrm{a}}}$ & $E_{\mu_{\mathrm{s}}}$ \\
\hline $0.07-0.4$ & $2-24$ & 6.2 & 24.5 & 2.8 & 13.4 \\
$0.07-0.4$ & $1-12$ & 5.9 & 57.1 & 1.8 & 19.6 \\
$0.07-0.4$ & $0.5-6$ & 4.8 & 106.2 & 3.3 & 25.5 \\
$0.07-0.4$ & $0.25-3$ & 3.9 & 156.0 & 4.3 & 34.8 \\
$0.07-0.4$ & $0.1-1.2$ & 3.8 & 329.4 & 3.2 & 47.4 \\
$0.14-0.8$ & $2-24$ & 4.7 & 18.9 & 3.7 & 13.4 \\
$0.035-0.2$ & $0.5-6$ & 4.3 & 124.6 & 2.2 & 29.4 \\
$0.0175-0.1$ & $0.25-3$ & 3.1 & 217.0 & 1.3 & 46.0 \\
$0.007-0.04$ & $0.1-1.2$ & 2.0 & 333.0 & 1.0 & 58.7 \\
\hline
\end{tabular}

the DA can not be considered to be accurate approximation. Thus, the Gaussian approximation of the modeling error may not be accurate, and consequently the Bayesian approximation error method is unable to compensate the errors of the absorption coefficient. By contrast, in the case where the values of absorption coefficient also decrease, the difference between the DA and Monte Carlo model is smaller and thus the BAE is able to compensate the modeling errors of the DA better.

Decreasing scattering coefficient of the simulated distribution while keeping the absorption coefficient constant should, in theory, decrease the accuracy of the estimates due to the increased modeling errors of the DA. While this effect is observed in the scattering estimates, the absorption estimates are more accurate when the scattering decreases. We expect this to be due to the fact, that lower scattering coefficient results in increased number of photons near the center of the domain, thus increasing the signal-to-noise ratio of the data. Likewise, decreasing both scattering or absorption coefficient increases the signal-to-noise ratio in greater extent, which can be especially seen in the situation where both scattering, and absorption coefficient are low. However, due to the ill-posedness of the estimation of the scattering coefficient, this does not result in more accurate scattering estimates.

With 5\% noise level, the relative errors of the MAP-EEM scattering estimates are smaller compared to the MAP-CEM estimates, but this difference is not as clear as with lower amount of additive noise. Further, the relative errors of MAP-CEM and MAP-EEM absorption estimates are close to each other in all ranges of the optical parameters. In this situation, the modeling errors of the DA are most likely significantly smaller than the random measurement noise and the effect of the modeling errors can be regarded as insignificant. For example, with $0.1 \%$ noise level the mean of the ratio $\operatorname{trace}\left(\Gamma_{\varepsilon}\right) / \operatorname{trace}\left(\Gamma_{\mathrm{e}}\right)$ is 30.8 , whereas with $5 \%$ noise level the ratio is 0.62 .

\subsection{Blood-vessel-mimicking inclusions}

The simulated optical parameters and the MAP estimates with the blood-vessel-mimicking inclusions are shown in Figure 9 Further, the MAP-estimates with credibility intervals along the cross-section through the domain are shown in Figure 10 and marginal densities of the posterior distributions in two points inside the domain are presented in the Figure 11.

Similarly as in the smooth inclusions, the absorption estimates appear similar in visual inspection, but the MAP-CEM estimate has more systematic error and artefacts which can be 

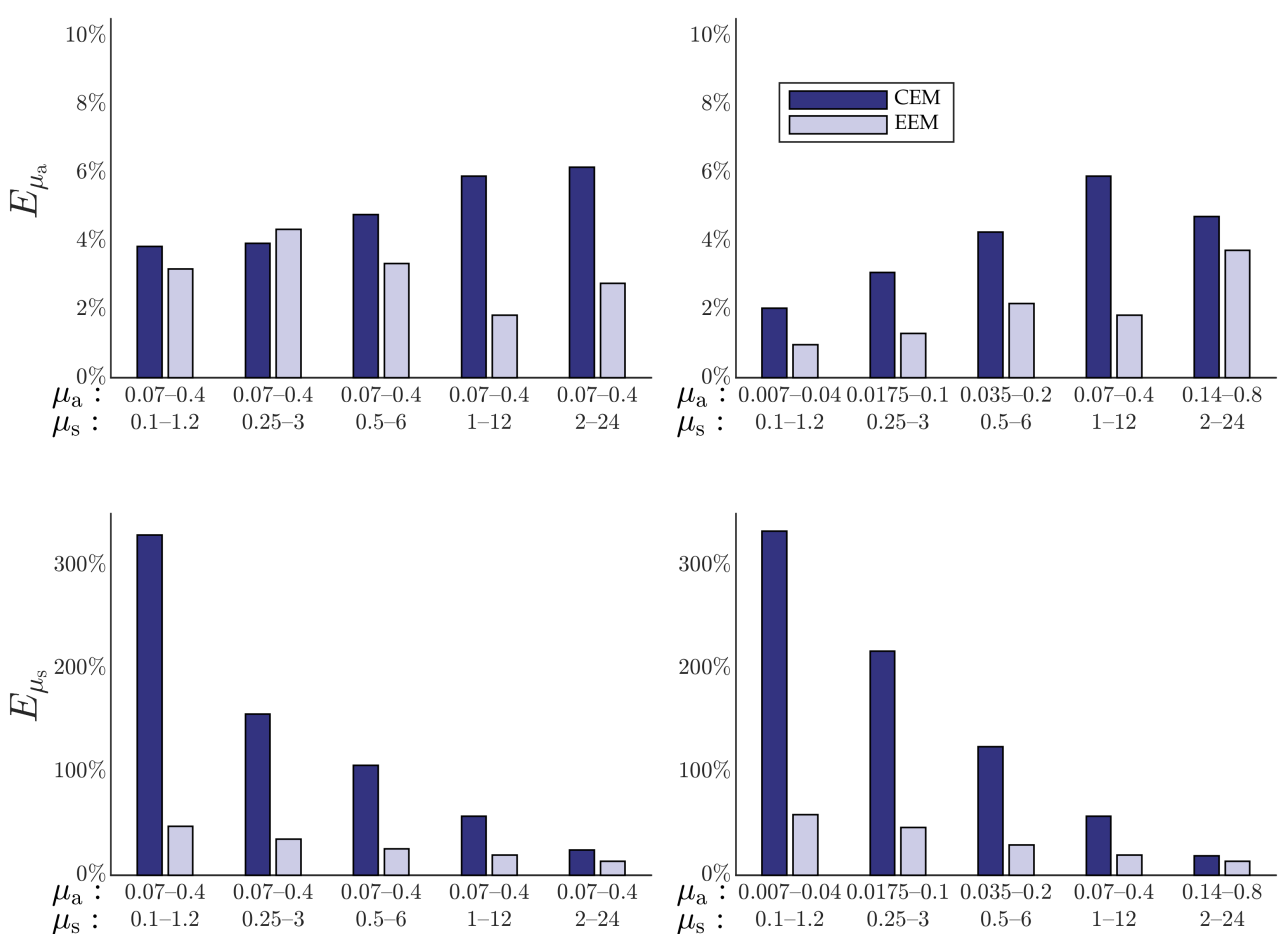

Figure 6: Relative errors $E_{\mu_{\mathrm{a}}}$ and $E_{\mu_{\mathrm{s}}}$ of the MAP-CEM (dark blue) and MAP-EEM (light blue) estimates (Table 4) with different ranges of optical parameters with $0.1 \%$ random noise. In the first column only the range of scattering coefficient varied, and in the second column range of both absorption and scattering coefficient varied. First row represents the absorption coefficient and second row scattering coefficient. The horizontal axis corresponds to different ranges of the optical parameter values.

Table 5: Relative errors of the MAP-CEM and MAP-EEM estimates $E_{\mu_{\mathrm{a}}}(\%)$ and $E_{\mu_{\mathrm{s}}}(\%)$ with different ranges of absorption $\mu_{\mathrm{a}}\left(\mathrm{mm}^{-1}\right)$ and scattering $\mu_{\mathrm{s}}\left(\mathrm{mm}^{-1}\right)$ coefficients with $1 \%$ noise.

\begin{tabular}{cccccc}
\hline & & \multicolumn{2}{c}{ MAP-CEM } & \multicolumn{2}{c}{ MAP-EEM } \\
\hline$\mu_{\mathrm{a}}$ range & $\mu_{\mathrm{s}}$ range & $E_{\mu_{\mathrm{a}}}$ & $E_{\mu_{\mathrm{s}}}$ & $E_{\mu_{\mathrm{a}}}$ & $E_{\mu_{\mathrm{s}}}$ \\
\hline $0.07-0.4$ & $2-24$ & 4.7 & 20.5 & 2.7 & 16.3 \\
$0.07-0.4$ & $1-12$ & 6.2 & 37.2 & 2.6 & 20.3 \\
$0.07-0.4$ & $0.5-6$ & 4.2 & 66.2 & 3.7 & 27.8 \\
$0.07-0.4$ & $0.25-3$ & 3.9 & 113.5 & 3.8 & 31.8 \\
$0.07-0.4$ & $0.1-1.2$ & 3.9 & 247.4 & 3.6 & 41.4 \\
$0.14-0.8$ & $2-24$ & 9.4 & 24.0 & 8.2 & 20.9 \\
$0.035-0.2$ & $0.5-6$ & 4.2 & 75.1 & 2.4 & 26.6 \\
$0.0175-0.1$ & $0.25-3$ & 3.2 & 128.4 & 1.8 & 33.2 \\
$0.007-0.04$ & $0.1-1.2$ & 2.7 & 269.6 & 1.4 & 49.1 \\
\hline
\end{tabular}



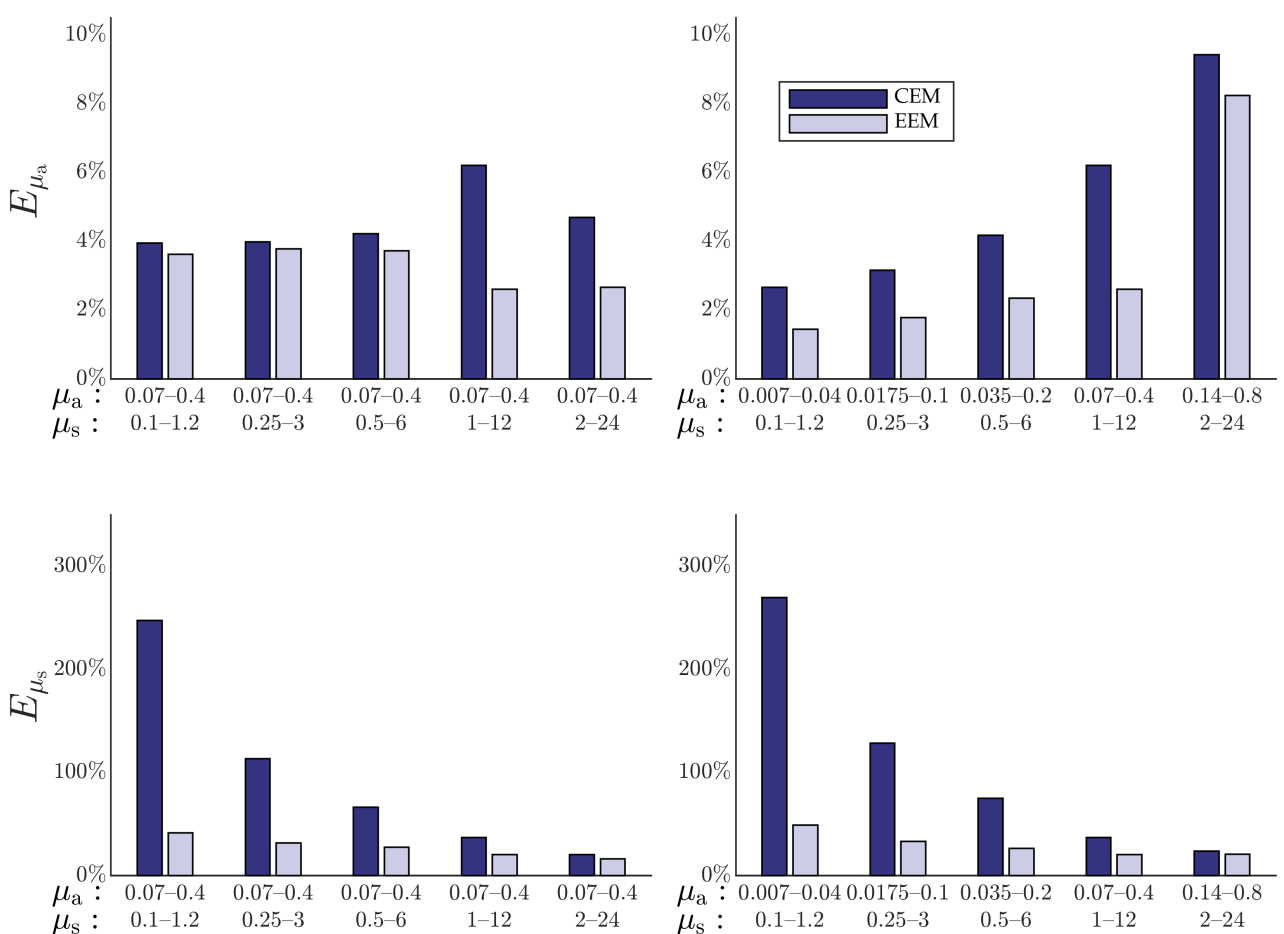

Figure 7: Relative errors $E_{\mu_{\mathrm{a}}}$ and $E_{\mu_{\mathrm{s}}}$ of the MAP-CEM (dark blue) and MAP-EEM (light blue) estimates (Table 5) with different ranges of optical parameters with $1 \%$ random noise. In the first column only the range of scattering coefficient varied, and in the second column range of both absorption and scattering coefficient varied. First row represents the absorption coefficient and second row scattering coefficient. The horizontal axis corresponds to different ranges of the optical parameter values.

Table 6: Relative errors of the MAP-CEM and MAP-EEM estimates $E_{\mu_{\mathrm{a}}}(\%)$ and $E_{\mu_{\mathrm{s}}}(\%)$ with different ranges of absorption $\mu_{\mathrm{a}}\left(\mathrm{mm}^{-1}\right)$ and scattering $\mu_{\mathrm{s}}\left(\mathrm{mm}^{-1}\right)$ coefficients with $5 \%$ noise.

\begin{tabular}{cccccc}
\hline & & \multicolumn{2}{c}{ MAP-CEM } & \multicolumn{2}{c}{ MAP-EEM } \\
\hline$\mu_{\mathrm{a}}$ range & $\mu_{\mathrm{s}}$ range & $E_{\mu_{\mathrm{a}}}$ & $E_{\mu_{\mathrm{s}}}$ & $E_{\mu_{\mathrm{a}}}$ & $E_{\mu_{\mathrm{s}}}$ \\
\hline $0.07-0.4$ & $2-24$ & 9.6 & 31.0 & 10.8 & 29.6 \\
$0.07-0.4$ & $1-12$ & 7.4 & 33.0 & 7.5 & 26.9 \\
$0.07-0.4$ & $0.5-6$ & 7.3 & 52.1 & 6.6 & 27.8 \\
$0.07-0.4$ & $0.25-3$ & 6.5 & 86.7 & 8.5 & 32.7 \\
$0.07-0.4$ & $0.1-1.2$ & 6.8 & 159.8 & 9.6 & 72.6 \\
$0.14-0.8$ & $2-24$ & 21.6 & 34.8 & 21.6 & 34.5 \\
$0.035-0.2$ & $0.5-6$ & 6.6 & 51.9 & 6.0 & 24.6 \\
$0.0175-0.1$ & $0.25-3$ & 5.8 & 91.7 & 5.4 & 38.0 \\
$0.007-0.04$ & $0.1-1.2$ & 5.6 & 186.7 & 5.1 & 47.6 \\
\hline
\end{tabular}



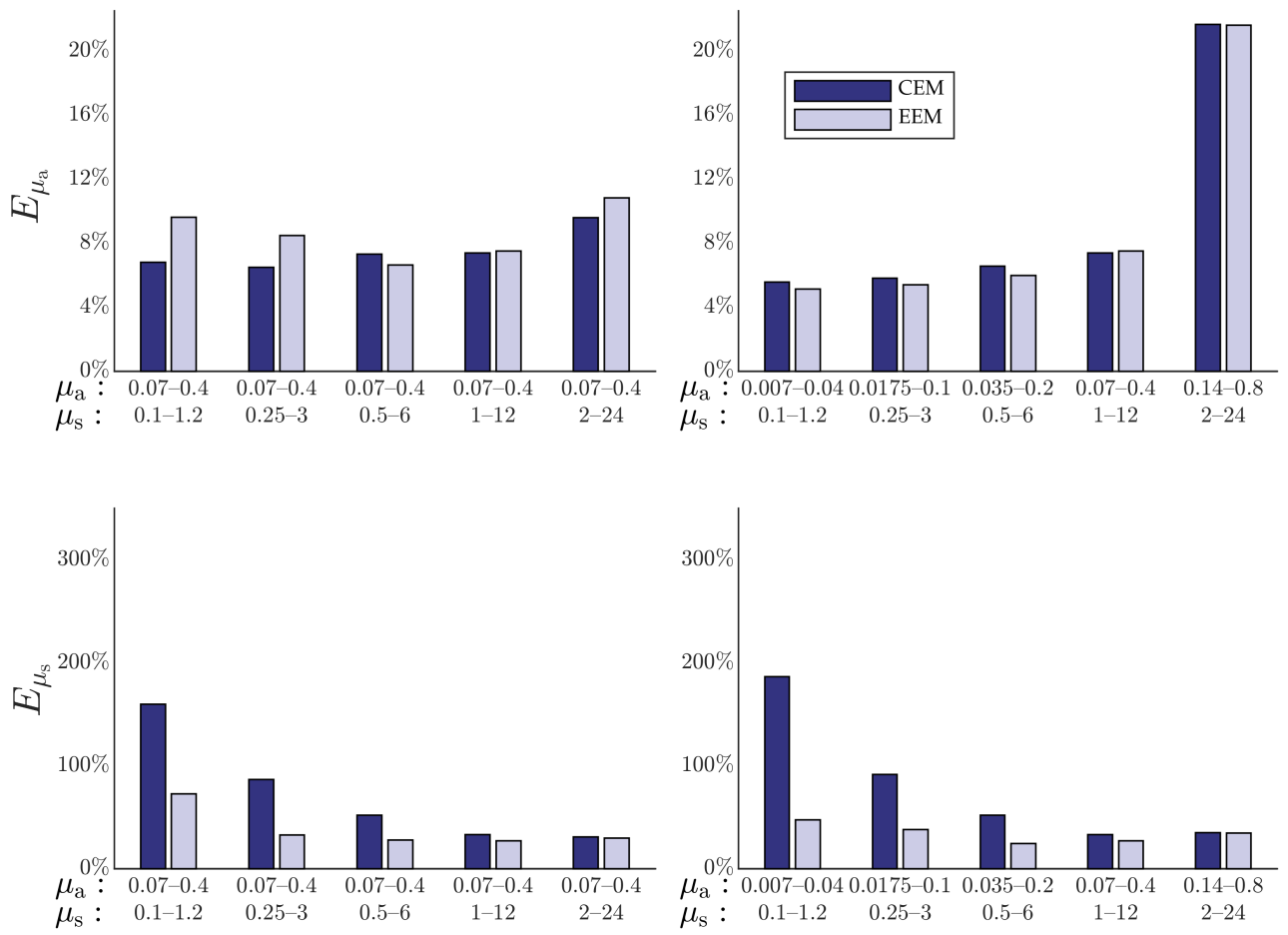

Figure 8: Relative errors $E_{\mu_{\mathrm{a}}}$ and $E_{\mu_{\mathrm{s}}}$ of the MAP-CEM (dark blue) and MAP-EEM (light blue) estimates (Table 6 with different ranges of optical parameters with 5\% random noise. In the first column only the range of scattering coefficient varied, and in the second column range of both absorption and scattering coefficient varied. First row represents the absorption coefficient and second row scattering coefficient. The horizontal axis corresponds to different ranges of the optical parameter values. 

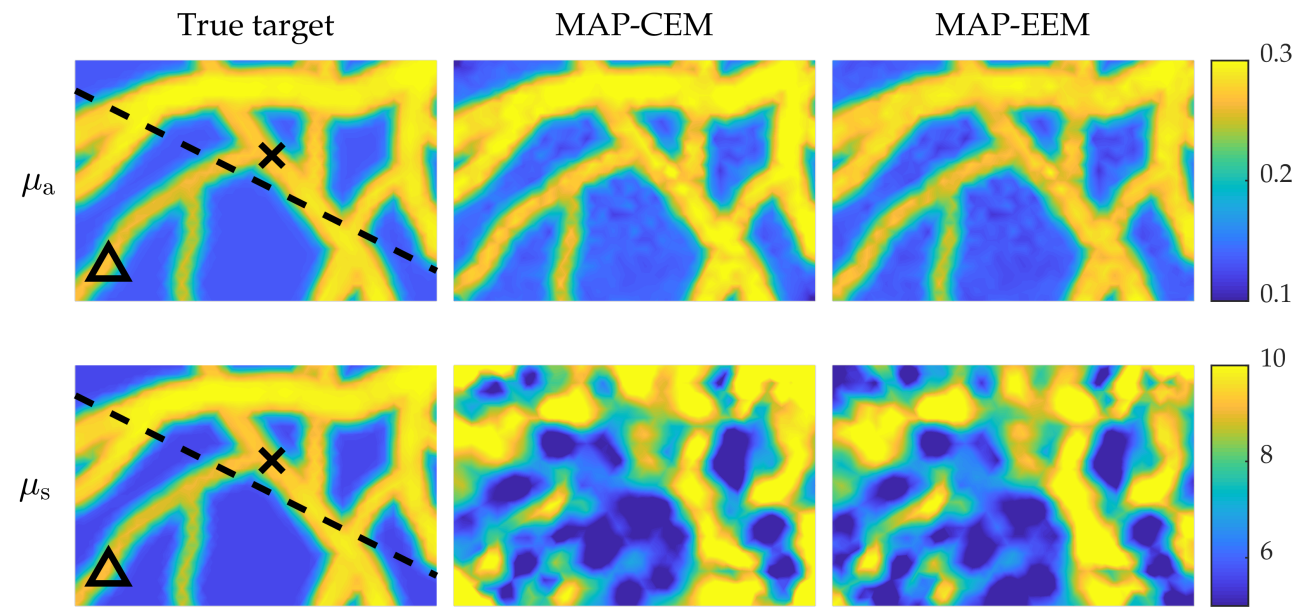

Figure 9: True target (first column), MAP estimate with conventional error model (second column) and MAP estimates with enhanced error model (third column). The black dashed line indicates the cross-section in which the credibility intervals are plotted. First row represent absorption $\mu_{\mathrm{a}}\left(\mathrm{mm}^{-1}\right)$ and second row scattering $\mu_{\mathrm{s}}\left(\mathrm{mm}^{-1}\right)$ coefficient.

Table 7: Relative errors of the MAP-CEM and MAP-EEM estimates $E_{\mu_{\mathrm{a}}}(\%)$ and $E_{\mu_{\mathrm{s}}}(\%)$ with blood-vessel-mimicking inclusions.

\begin{tabular}{cccc}
\hline \multicolumn{2}{c}{ MAP-CEM } & \multicolumn{2}{c}{ MAP-EEM } \\
\hline$E_{\mu_{\mathrm{a}}}$ & $E_{\mu_{\mathrm{s}}}$ & $E_{\mu_{\mathrm{a}}}$ & $E_{\mu_{\mathrm{s}}}$ \\
\hline $4.8 \%$ & $22.0 \%$ & $4.1 \%$ & $15.0 \%$ \\
\hline
\end{tabular}

especially seen in the areas of low absorption near the center of the domain. Likewise, even though neither of the scattering estimates accurately resemble the true scattering distribution, the MAP-CEM scattering estimates have more artifacts compared to the MAP-EEM estimate, especially near the boundaries of the domain. This difference is also evident when comparing the relative errors, which are presented in Table 7. However, the differences between the MAPCEM and MAP-EEM estimates are smaller compared to the smooth inclusions. This could be probably improved by using more accurate teaching distribution for the BAE approach and prior distribution in the solution of the inverse problem.

Inspecting the credibility intervals and the marginal distributions shows that in some areas of the domain the MAP-EEM absorption estimates are closer to the true values than the MAP-CEM estimates. However, in some areas the BAE method overcompensates the modeling errors and the MAP-EEM absorption estimate is lower than the true value. Furthermore, the credibility intervals of the MAP-EEM cover the true values more reliably compared to the MAP-CEM estimates, especially near the boundaries of the domain.

\section{Discussion and conclusions}

In this work, compensation of modeling errors of the DA utilizing the Bayesian approximation error method in QPAT was studied. The BAE model was trained using Monte Carlo 

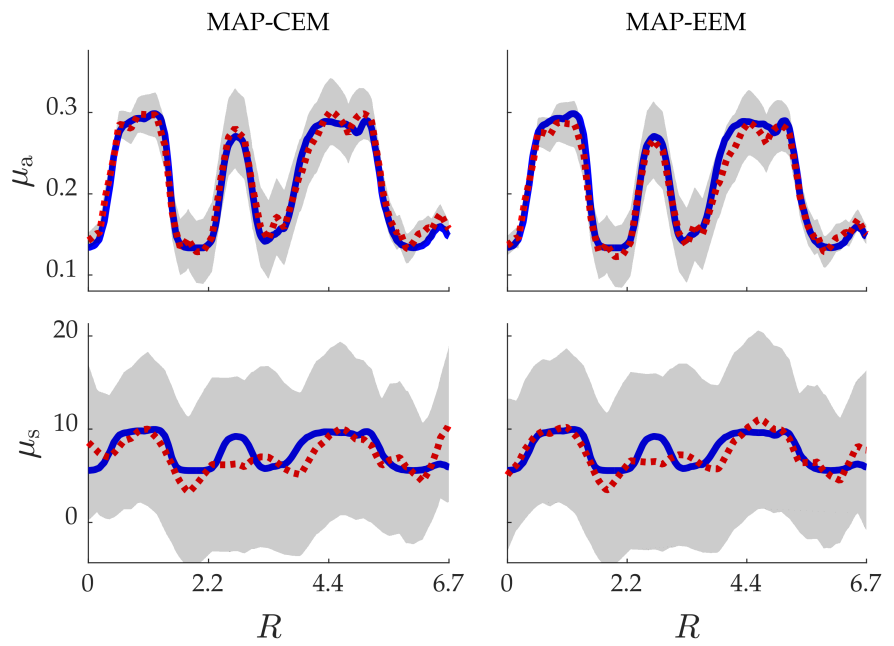

Figure 10: MAP-CEM reconstructions (first column) and MAP-EEM reconstructions (second column) with credibility intervals of the simulations with blood-vessel mimicking simulations along the cross-section shown in Figure 9 Solid blue line is the true value and dotted red line is MAP estimate and gray area covers the credibility interval $\left[\hat{\mu}-3 \sigma_{\hat{\mu}}, \hat{\mu}+\right.$ $\left.3 \sigma_{\hat{\mu}}\right]$. First row presents the absorption $\mu_{\mathrm{a}}\left(\mathrm{mm}^{-1}\right)$ and second row the scattering $\mu_{\mathrm{s}}\left(\mathrm{mm}^{-1}\right)$. Horizontal axis $R$ presents the distance from the upper left end point of the cross-section.
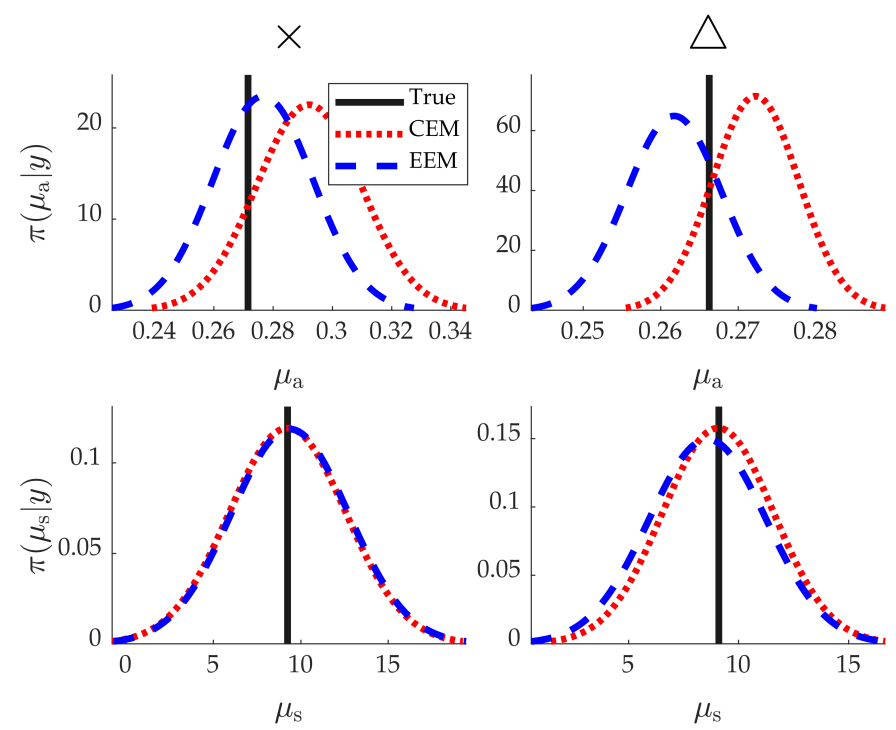

Figure 11: Marginal probability densities of the posterior distributions with blood-vessel-mimicking inclusions. True value (solid vertical line), the approximation of the posterior distribution of MAP-CEM reconstructions (red dotted line) and MAP-EEM reconstructions (blue dashed line). The first row presented the absorption $\mu_{\mathrm{a}}\left(\mathrm{mm}^{-1}\right)$ and second row the scattering $\mu_{\mathrm{s}}\left(\mathrm{mm}^{-1}\right)$. First column presents the results in the point marked with $\times$ and second row with $\Delta$ in the Figure 9 
simulations. The approach was studied with numerical simulations with various ranges of optical parameters and different noise levels. As the simulations demonstrate, utilizing the BAE method increased the accuracy of the estimates when the level of measurement noise was $0.1 \%$ or $1 \%$. The effect was visible when comparing the results visually, and also when comparing the relative errors of the estimates. While the DA can be considered as sufficiently accurate model in the reference range of optical parameters in this study, the small size of the domain introduces modeling errors. Some differences in the model solutions are also due to the discretization of the problem that affects especially on the fluence predictions of the FE-based diffusion model.

In addition to improving the quality of the images and accuracy of the estimates, utilizing the BAE modeling increased the standard deviation of the posterior approximation of the estimates. This resulted in wider credibility intervals of the estimates, which in this work covered the true target parameter values more reliably. Therefore, the credibility estimates of the MAPEEM estimates can be seen as more reliable to assess the uncertainty of the estimate. This was especially evident in the absorption estimates, where the MAP-CEM estimates resemble the true distribution by visual inspection and appear to be qualitatively accurate, but the small difference that is apparent between the true and estimated values is not covered by the credibility interval in the whole domain. In the corresponding results utilizing the BAE modeling, the MAP estimates were more accurate and the true values were within the credible interval.

A Monte Carlo model was utilized in the BAE modeling as an accurate model for the light transport. The stochastic nature of Monte Carlo means that additional noise was introduced to the data simulation and approximation error sampling. In order to minimize this noise, number of photon packets was set high and thus, the effect of this noise was insignificant compared to the additive random noise. Furthermore, in this work the Monte Carlo model utilized different basis functions compared to the DA model. In addition to the modeling errors, this introduces numerical errors between the models which the BAE modeling compensates.

In this study, the inclusions were quite simple. Reconstructing more complex structures would require finer discretizations which would increase the computational cost of the algorithm significantly. This would require further development of methods for model reduction in QPAT. Further, in practical applications, the first step of QPAT would be the reconstruction of the initial pressure distribution and the absorbed optical energy density from the acoustic boundary measurements. Thus, the image reconstruction problem studied in this work is only a part of the full process. Furthermore, in this work the domain was illuminated from all sides which may not be possible in clinical applications. The future studies therefore include, in addition to the above mentioned model reduction, considering more realistic imaging situations with the full QPAT model.

In conclusion, the Bayesian approach in QPAT can provide accurate absorption and scattering estimates with credibility estimates. Furthermore, the Bayesian approximation error modeling can compensate modeling errors of approximative forward model on a broad range of optical parameters. Further, modeling of the errors can improve the reliability of the credibility intervals.

\section{Acknowledgements}

This study was funded by Academy of Finland (projects 314411 and 312342 Finnish Centre of Excellence in Inverse Modelling and Imaging), Jane and Aatos Erkko Foundation, Instrumentarium Science Foundation and Antti and Jenny Wihuri Foundation. In addition, the authors wish to acknowledge CSC - IT Center for Science, Finland, for computational resources. 


\section{References}

[1] P. Beard, Biomedical photoacoustic imaging, Interface Focus 1 (2011) 602-631.

[2] J. Xia, J. Yao, L. V. Wang, Photoacoustic tomography: principles and advances, Electromagn Waves 147 (2014) $1-22$.

[3] L. V. Wang (Ed.), Photoacoustic Imaging and Spectroscopy, CRC Press, 2009.

[4] M. Xu, L. V. Wang, Photoacoustic imaging in biomedicine, Rev Sci Instrum 77 (2006) 041101.

[5] C. Li, L. V. Wang, Photoacoustic tomography and sensing in biomedicine, Phys Med Biol 54 (2009) R59-R97.

[6] L. V. Wang, Prospects of photoacoustic tomography, Med Phys 35 (12) (2008) 5758-5767.

[7] J. Yao, J. Xia, K. I. Maslov, M. Nasiriavanaki, V. Tsytsarev, A. V. Demchenko, L. V. Wang, Noninvasive photoacoustic computed tomography of mouse brain metabolism in vivo, Neuroimage 64 (2013) $257-266$.

[8] B. Cox, J. G. Laufer, S. R. Arridge, P. C. Beard, Quantitative spectroscopic photoacoustic imaging: a review, J Biomed Opt 17 (6) (2012) 061202.

[9] A. Pulkkinen, B. T. Cox, S. R. Arridge, J. P. Kaipio, T. Tarvainen, A Bayesian approach to spectral quantitative photoacoustic tomography, Inv Probl 30 (2014) 065012.

[10] G. Bal, K. Ren, On multi-spectral quantitative photoacoustic tomography in a diffusive regime, Inv Probl 28 (2012) 025010

[11] B. T. Cox, S. R., Arridge, P. C. Beard, Estimating chromophore distributions from multiwavelength photoacoustic images, J Opt Soc Am A 26 (2) (2009) 443-455.

[12] J. Laufer, B. Cox, E. Zhang, P. Beard, Quantitative determination of chromophore concentrations from 2D photoacoustic images using a nonlinear model-based inversion scheme, Appl Opt 49 (8) (2010) 1219-1233.

[13] D. Razansky, J. Baeten, V. Ntziachristos, Sensitivity of molecular target detection by multispectral optoacoustic tomography (MSOT), Med Phys 36 (3) (2009) 939-945.

[14] G. S. Alberti, H. Ammari, Disjoint sparsity for signal separation and applications to hybrid inverse problems in medical imaging, Appl Comput Harmon Anal 42 (2) (2017) 319 - 349.

[15] B. Banerjee, S. Bagchi, R. M. Vasu, D. Roy, Quantitative photoacoustic tomography from boundary pressure measurements: noniterative recovery of optical absorption coefficient from the reconstructed absorbed energy map, J Opt Soc Am A 25 (9) (2008) 2347-2356.

[16] T. Tarvainen, B. T. Cox, J. P. Kaipio, S. R. Arridge, Reconstructing absorption and scattering distributions in quantitative photoacoustic tomography, Inv Probl 28 (2012) 084009.

[17] A. Pulkkinen, V. Kolehmainen, J. P. Kaipio, B. T. Cox, S. R. Arridge, T. Tarvainen, Approximate marginalization of unknown scattering in quantitative photoacoustic tomography, Inv Probl Imag 8 (3) (2014) 811-829.

[18] A. V. Mamonov, K. Ren, Quantitative photoacoustic imaging in radiative transport regime, Commun Math Sci 12 (2014) 201-234.

[19] G. Bal, K. Ren, Multi-source quantitative photoacoustic tomography in a diffusive regime, Inv Probl 27 (2011) 075003 .

[20] T. Saratoon, T. Tarvainen, B. T. Cox, S. R. Arridge, A gradient-based method for quantitative photoacoustic tomography using the radiative transfer equation, Inv Probl 29 (2013) 075006.

[21] A. D. Cezaro, F. T. D. Cezaro, J. S. Suarez, Regularization approaches for quantitative photoacoustic tomography using the radiative transfer equation, J Math Anal Appl 429 (2015) 415-438.

[22] P. Shao, T. Harrison, R. J. Zemp, Iterative algorithm for multiple illumination photoacoustic tomography (MIPAT) using ultrasound channel data, Biomed Opt Express 3 (12) (2012) 3240-3248.

[23] W. Naetar, O. Scherzer, Quantitative photoacoustic tomography with piecewise constant material parameters, SIAM J Imaging Sci 7 (3) (2014) 1755-1774.

[24] A. Pulkkinen, B. T. Cox, S. R. Arridge, H. Goh, J. P. Kaipio, T. Tarvainen, Direct estimation of optical parameters from photoacoustic time series in quantitative photoacoustic tomography, IEEE Trans Med Imag 35 (11) (2016) 2497-2508.

[25] T. Ding, K. Ren, S. Vallélian, A one-step reconstruction algorithm for quantitative photoacoustic imaging, Inv Probl $31(2015) 095005$

[26] N. Song, C. Deumié, A. D. Silva, Considering sources and detectors distributions for quantitative photoacoustic tomography, Biomed Opt Express 5 (11) (2014) 3960-3974.

[27] M. Haltmeier, L. Neumann, S. Rabanser, Single-stage reconstruction algorithm for quantitative photoacoustic tomography, Inv Probl 31 (2015) 065005.

[28] H. Gao, J. Feng, L. Song, Limited-view multi-source quantitative photoacoustic tomography, Inv Probl 31 (2015) 065004 .

[29] A. Javaherian, S. Holman, Direct quantitative photoacoustic tomography for realistic acoustic media, Inv Probl 35 (8) (2019) 084004.

[30] A. Ishimaru, Wave Propagation and Scattering in Random Media, Vol. 1, Academic Press, New York, 1978

[31] S. A. Prahl, A Monte Carlo model of light propagation in tissue, in: G. J. Mueller, D. H. Sliney, R. F. Potter (Eds.), 
Dosimetry of Laser Radiation in Medicine and Biology, Vol. 10305, International Society for Optics and Photonics, SPIE, 1989, pp. $105-114$.

[32] C. Zhu, Q. Liu, Review of Monte Carlo modeling of light transport in tissues, J Biomed Opt 18 (5) (2013) 1 - 13 13.

[33] J. Buchmann, B. A. Kaplan, S. Powell, S. Prohaska, J. Laufer, Three-dimensional quantitative photoacoustic tomography using an adjoint radiance Monte Carlo model and gradient descent, J Biomed Opt 24 (6) (2019) 1 13.

[34] L. Yao, Y. Sun, H. Jiang, Transport-based quantitative photoacoustic tomography: simulations and experiments, Phys Med Biol 55 (2010) 1917-1934.

[35] R. Hochuli, S. Powell, S. Arridge, B. Cox, Quantitative photoacoustic tomography using forward and adjoint Monte Carlo models of radiance, J Biomed Opt 21 (12) (2016) 126004.

[36] S. Okawa, T. Hirasawa, K. Tsujita, T. Kushibiki, M. Ishihara, 3D quantitative photoacoustic image reconstruction using Monte Carlo method and linearization 10494 (2018).

[37] A. Leino, T. Lunttila, M. Mozumder, A. Pulkkinen, T. Tarvainen, Perturbation Monte Carlo method for quantitative photoacoustic tomography, IEEE Trans Med Imag, Submitted.

[38] J. Buchmann, B. Kaplan, S. Powell, S. Prohaska, J. Laufer, Quantitative pa tomography of high resolution 3-d images: Experimental validation in a tissue phantom, Photoacoustics 17 (2020) 100157.

[39] H. Gao, L. Phan, Y. Lin, Parallel multigrid solver of radiative transfer equation for photon transport via graphics processing unit, J Biomed Opt 17 (9) (2012) 096004.

[40] M. Badri, P. Jolivet, B. Rousseau, Y. Favennec, Preconditioned Krylov subspace methods for solving radiative transfer problems with scattering and reflection, Comput Math Appl 77 (6) (2019) 1453 - 1465.

[41] J. Jia, H. K. Kim, A. H. Hielscher, Fast linear solver for radiative transport equation with multiple right hand sides in diffuse optical tomography, J Quant Spectrosc Radiat Transf 167 (2015).

[42] S. R. Arridge, Optical tomography in medical imaging, Inv Probl 15 (1999) R41-R93.

[43] C. Frederick, K. Ren, S. Vallelian, Image reconstruction in quantitative photoacoustic tomography with the simplified p_2 approximation, SIAM J Imaging Sci 11 (2018) 2847-2876.

[44] T. Tarvainen, V. Kolehmainen, A. Pulkkinen, M. Vauhkonen, M. Schweiger, S. R Arridge, J. Kaipio, Approximation error approach for compensating modelling errors between the radiative transfer equation and the diffusion approximation in diffuse optical tomography, Inv Probl 26 (2009) 015005.

[45] L. Yao, Y. Sun, H. Jiang, Quantitative photoacoustic tomography based on the radiative transfer equation, Opt Lett 34 (12) (2009) 1765-1767.

[46] K. Ren, G. Bal, A. H. Hielscher, Transport- and diffusion-based optical tomography in small domains: a comparative study, Appl Opt 46 (27) (2007) 6669-6679.

[47] N. Hänninen, A. Pulkkinen, T. Tarvainen, Image reconstruction with reliability assessment in quantitative photoacoustic tomography, J Imaging 4 (12) (2018).

[48] T. Tarvainen, A. Pulkkinen, B. T. Cox, J. P. Kaipio, S. R. Arridge, Bayesian image reconstruction in quantitative photoacoustic tomography, IEEE Trans Med Imag 32 (12) (2013) 2287-2298.

[49] T. Tarvainen, V. Kolehmainen, J. P. Kaipio, S. R. Arridge, Corrections to linear methods for diffuse optical tomography using approximation error modelling, Biomed. Opt. Express 1 (1) (2010) 209-222.

[50] T. Tarvainen, M. Vauhkonen, V. Kolehmainen, S. R. Arridge, J. P. Kaipio, Coupled radiative transfer equation and diffusion approximation model for photon migration in turbid medium with low-scattering and non-scattering regions, Phys Med Biol 50 (2005) 4913-4930.

[51] O. Lehtikangas, T. Tarvainen, A. Kim, S. Arridge, Finite element approximation of the radiative transport equation in a medium with piece-wise constant refractive index, J Comput Phys 282 (2015) 345 - 359.

[52] A. A. Leino, A. Pulkkinen, T. Tarvainen, ValoMC: a Monte Carlo software and MATLAB toolbox for simulating light transport in biological tissue, OSA Continuum 2 (3) (2019) 957-972.

[53] J. Kaipio, E. Somersalo, Statistical and Computational Inverse Problems, Springer, New York, 2005.

[54] A. Nissinen, V. Kolehmainen, J. P. Kaipio, Compensation of modelling errors due to unknown domain boundary in electrical impedance tomography, IEEE Trans Med Imag 30 (2010) 231-242.

[55] J. Koponen, T. Huttunen, T. Tarvainen, J. Kaipio, Approximation error method for full-wave tomography, J Acoust Soc Am 133 (5) (2013) 3230-3230.

[56] J. Heino, E. Somersalo, J. P. Kaipio, Compensation for geometric mismodelling by anisotropies in optical tomography, Opt Express 13 (1) (2005) 296-308.

[57] S. R. Arridge, J. P. Kaipio, V. Kolehmainen, M. Schweiger, E. Somersalo, T. Tarvainen, M. Vauhkonen, Approximation errors and model reduction with an application in optical diffusion tomography, Inv Probl 22 (2006) $175-195$.

[58] W. F. Cheong, S. A. Prahl, A. J. Welch, A review of the optical properties of biological tissues, IEEE J Quantum Electron 26 (1990) 2166-2185.

[59] S. L. Jacques, Optical properties of biological tissues: a review, Phys Med Biol 58 (2013). 
[60] A. N. Bashkatov, E. A. Genina, V. V. Tuchin, Optical properties of skin, subcutaneous, and muscle tissues: a review, J Innov Opt Health Sci 4 (1) (2011) 9-38.

[61] C. E. Rasmussen, C. K. I. Williams, Gaussian Processes for Machine Learning, MIT Press, 2006. 